\section{ECCOMAS}

\section{Proceedia}

UNCECOMP 2021

$4^{\text {th }}$ ECCOMAS Thematic Conference on Uncertainty Quantification in Computational Sciences and Engineering M. Papadrakakis, V. Papadopoulos, G. Stefanou (eds.) Streamed from Athens, Greece, 28 -30 June 2021

\title{
NUMERICAL SIMULATION OF A 3D PRINTED WALL STRUCTURE DURING THE PROCESS OF PRINTING CONSIDERING UNCERTAINTY
}

\author{
Meron Mengesha ${ }^{2}$, Albrecht Schmidt ${ }^{1,2}$, Luise Göbel ${ }^{1}$, \\ Tom Lahmer ${ }^{1,2}$, and Carsten Könke ${ }^{1}$ \\ ${ }^{1}$ Materials Research and Testing Institute (MFPA) at the Bauhaus-Universität Weimar, Germany \\ e-mail: meron.wondafrash@gmail.com \\ ${ }^{2}$ Institute of Structural Mechanics, Bauhaus-Universität Weimar, Germany
}

\begin{abstract}
$3 D$ concrete printing technology is getting increasing recognition in the construction industry. The extrusion-based printing method represents the most popular and promising one among the $3 D$ printing techniques for concrete. However, mostly time-consuming trial-anderror explorations, i.e. mainly experimental studies have been performed so far.

By utilizing numerical simulation, a fundamental understanding of the relations between process - process parameters - product properties could be achieved. Also, they enable us to study the dependencies of properties of the printed product on process parameters and material behavior. The extrusion-based $3 D$ concrete printing process can be reliably controlled and optimized by taking into account the uncertain nature of the process and material parameters.

In this study, the Finite Element (FE) method combined with a pseudo-density approach, following the soft-killing approaches in topology optimization is applied. The numerical simulations allow to reliably estimate the strength-based failure mechanisms that might occur during the $3 D$ concrete printing of a wall structure by varying one of the printing process parameter printing velocity.
\end{abstract}

Keywords: 3D concrete printing, Uncertainty, SFEM simulation, Pseudo-density approach, Random Process, Reliability, Strength-based failure 


\section{INTRODUCTION}

Very recently, additive manufacturing techniques for concrete technology have gained wide attention. They indicated their potential to become a serious supplement to conventional concrete casting in molds. 3D concrete printing (3DCP) is one of the fastest evolving technologies in construction engineering, which is illustrated by the rapid growth of both research and industry projects carried out worldwide [1]. The main reason for this is it directly addresses the challenges related to the sustainability and productivity of the construction industry.

However, the current practice is based on the trial-and-error procedure, which makes the research of the 3DCP process expensive and time-consuming [2].

One of the reasons is that there exist significant knowledge gaps regarding the relations between the design, material, and process parameters. The quality of the fabricated product is significantly influenced by these parameters which also exhibit interdependency [3].

Therefore, it is of vital importance to establish a relation between the process parameters and the printed product to avoid unreliability and failure [1, 4]. By implementing a numerical simulation of the 3DCP process, a more fundamental understanding of the relations between the printing process, the process parameters, and the properties of the printed product could be achieved.

Since the technology is new, the deterministic approach, i.e. applying safety factors to account for the uncertainty of the system, may not be applicable. Accordingly, the Stochastic Finite Element Method (SFEM) incorporating the spatially varying pseudo-density approach is proposed to include the uncertain nature of the process and material parameters of the extrusion-based 3D concrete printing. Also in the numerical modeling along with the progressing printing process, a previously generated finite element (FE) mesh is activated layer by layer that includes all material parameters. These vary spatially and temporarily due to the time dependency of the curing process. The numerical simulations allow to reliably estimate strength-based failure mechanisms that might occur during the $3 \mathrm{D}$ concrete printing of a wall structure.

\section{STATE OF THE ART}

To obtain a stable and reliable printed product, two criteria have to be considered and controlled during the manufacturing: the overall failure probability and the geometrical dimensions of the single layers. Non-sufficient strength, stiffness, or stability may already cause the failure of the structure during the printing process. These properties are strongly dependent on the printing process parameters, e.g. printing velocity, temperature, nozzle diameter, as well as on the concrete mixture.

In this context, Van der Putten et al. [21] studied the effects of the linear printing speed and the time gap between two subsequent layers on the microstructure of printed concrete. Accordingly, the two parameters significantly influence the surface roughness, the compressive strength, and the interlayer bonding strength. Therefore, it is of vital importance to establish a relation between the process parameters and the mechanical properties of the printed product in order to avoid unreliability and failure $[1,4]$.

Structural reliability analysis aims at computing the probability of failure, by accounting for different sources of uncertainties. These include inherent randomness of the material or lack of data [10], geometrical imperfection, random loading [11], uncertainties due to human error, and adopted model [12]. For example, for printable concrete Wolfs et al. [4] showed that the mechanical characteristics of printable concrete are random in nature with different values of coefficient of variation minimum of $3.8 \%$ to a maximum of $23 \%$. 
Since it includes safety definition and uncertainties in the analysis the probability of failure is a more reliable and complete measure of safety [19].

To compute the failure probability, it is necessary to formulate a limit state function $\mathrm{g}(\mathrm{x})$ which, for instances can include the displacements, stresses, or strains where $\mathrm{X}$ is a vector of basic random variables, which describe the randomness in the geometry, material properties, and loading, etc. [ 15,19].

$$
P_{f}=\int_{g(x) \leq 0} f_{X}(x) d x
$$

where $f_{X}$ is the joint probability density function (PDF) of random vector $\mathrm{X}$ and $\mathrm{g}(\mathrm{x})$ is the limit state function, with $\mathrm{g}(\mathrm{x}) \leq 0$ denoting the failure domain and $\mathrm{g}(\mathrm{x})>0$ is the safe domain. Making its direct estimation for Eq. (1) is computationally expensive in the general case, but various approximation methods have been developed to evaluate the failure probability, here the method used is the Adaptive Kriging Monte Carlo Simulation (AK-MCS), Which saves the costly evaluation of the actual limit state function [15]. It is based on Monte Carlo Simulation (MCS) and Kriging meta-model [24], by using the Kriging meta-model to approximate the limit state function, which is then combined with MCS to evaluate the probability of failure [22].

The limit state function for the extrusion-based 3D printing processes of the wall is elastic buckling and plastic collapse. The elastic buckling mechanism reflects failure caused by a loss of geometrical stability, while plastic collapse is characterized by the maximum stress reaching the material yield strength [23].

The value of the yield strength is dependent on the type of failure criterion adopted for the printing material. Two representative failure criteria are pressure-dependent shear failure following the Mohr-Coulomb theory and compressive failure described by the maximal stress theory [23].

To determine the maximum shear stress developed as a result of the self-weight, deterministic FEM is typically restricted to average values of the input variables, it fails to consider the uncertainties and leads to a rough representation of reality $[10,13,14]$.

To account for the various uncertainties arising in the model description (geometry, material properties, or loading) encountered in engineering practice, researchers have been trying to extend the standard FEM into the Stochastic Finite Element Method (SFEM) by incorporating random variables into the mathematical and computational formulations $[10,12,15]$. It has been named the Random Finite Element Method (RFEM) and the Probabilistic Finite Element Method (PFEM) [10].

SFEM consists mainly of discretization of stochastic fields, a FEM analysis part, and estimation of system response statistics [16]. Different approaches are available for the discretization of a stochastic field, some of them are the midpoint method, the interpolation method, the local average method. Once the stochastic fields are generated, Monte Carlo simulation is the most straightforward method, since it only needs repeated execution of an existing deterministic solution by utilizing many realizations of the random variables $[16,18,19]$.

\subsection{Compressive failure}

Plastic collapse is reached when the compressive strength becomes lower than the vertical compressive stress. Wolfs et al. [4], experimentally determined the evolution of the compressive strength over the time of the curing process and have developed equations based on the average result for $3 \mathrm{D}$ printable concrete. 


$$
\sigma_{\mathrm{y}}(\mathrm{r}, \mathrm{t})=\sigma_{\mathrm{y}, 0}+\sigma_{\mathrm{y}, 1} \mathrm{t}_{\mathrm{r}}
$$

where $\sigma_{\mathrm{y}, 0}$ is the initial yield strength of the printable concrete at the moment it leaves the printing nozzle, $\sigma_{y, 1}$ is the gradient of temporal increment, and $t_{r}$ is the curing time at a specific location.

However, experimental variations were observed in a series of compression and shear tests conducted at different ages of the fresh concrete [4]. It is shown that the experimentally obtained results exhibit a large scatter with coefficients of variation ranging from 13 to $21 \%$ for the compressive strength. Furthermore, as indicated in Eq. (2) the experiments have revealed that the studied material properties increase linearly over time. From these findings, it is obvious that reliability-oriented modeling needs to account for both temporal changes and the randomness of the material parameters.

Random fields are more frequently applied in structural engineering analyses related to concrete. A parameter considering spatial variability is mainly determined by the mean, the variance, and the scale of fluctuation [28,31]. For concrete structures in some literature, the correlation properties are usually taken into account by utilizing exponential functions [30]. Bottenbruch et al. [29], have done a numerical investigation on spatial correlation of concrete and identified that it is significant along with the layers for pouring concrete in layers by slip forming.

Nonetheless, in most literature, the correlation length is assumed and these assumptions are not consistent with each other [32], the reason for this is the lack of available data [30,31]. The probability of failure can be underestimated resulting in an unconservative design if this value is ignored or implicitly assumed to be infinite [12,16,33,35]. To avoid this the modeling needs to account for the correlation length.

The compressive stress $\sigma_{\mathrm{p}}(\mathrm{r}, \mathrm{t})$ depends on the height of the wall $\mathrm{h}(\mathrm{r})$ to be printed. This compressive stress acting on any of the layers can be written as follows [8]:

$$
\sigma_{\mathrm{p}}(\mathrm{r})=\rho_{c} \mathrm{gh}(\mathrm{r})
$$

Where $\rho_{c}$ is the material density, $g$ is the acceleration of gravity. Similarly, Wolfs et al. [4] experimentally determined the density of the printable concrete.

\section{NUMERICAL MODELING OF 3D CONCRETE PRINTING}

During the extrusion process the rheological properties of the concrete change. The material should be flowable at the pumping and extruding stage and after deposition, it should gain rapid strength to have a stable shape and to carry the subsequent layers $[5,6]$.

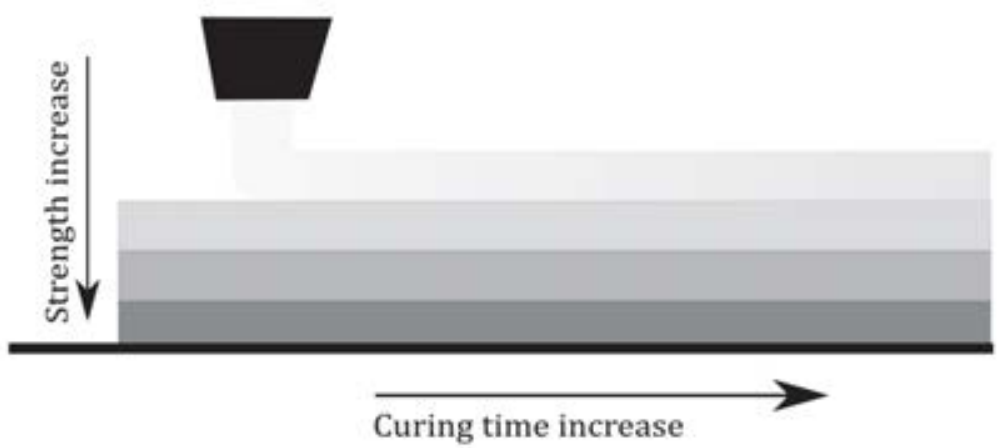

Figure 1: Gradient of the strength development of 3D printed concrete wall. 
To ensure the bond strength between layers the time gap between consecutive layers should be kept as low as possible [7]. This time dependency of the evolution of the yield stress is one of the main components in numerical modeling.

Fig. 1 shows the gradient of the concrete strength due to the different ages and resting times of the layers. Compared to the other layers, the concrete of the bottom layer is more mature than in the subsequent layers. To analyze the shape stability or buildability of the printed structure at a given time, this gradient of the strength over the height of the structure should be considered [8]. As each layer is activated in the numerical modeling this spatial variation is considered.

For each of the printed layer, the curing time $\left(t_{c}\right)$ can be calculated based on the printing velocity $\left(v_{p}\right)$, counting the number of layers from bottom to top according to the printing progress and assuming that there is no time gap between layers, the $i^{\text {th }}$ layer will have a curing time of:

$$
t_{c i}=(-i+(N+1)) \frac{L}{v_{p}},
$$

where $\mathrm{N}$ is the total number of layers and $\mathrm{L}$ is the length of the printed layer.

The numerical model is based on the finite element method (FEM) following the layerwise production process, i.e. each layer is activated separately. However, the problem arises when the layer thickness of the concrete and the size of the FE mesh do not coincide because the size of the FE is much lower than the thickness of the layers, see Fig.2.

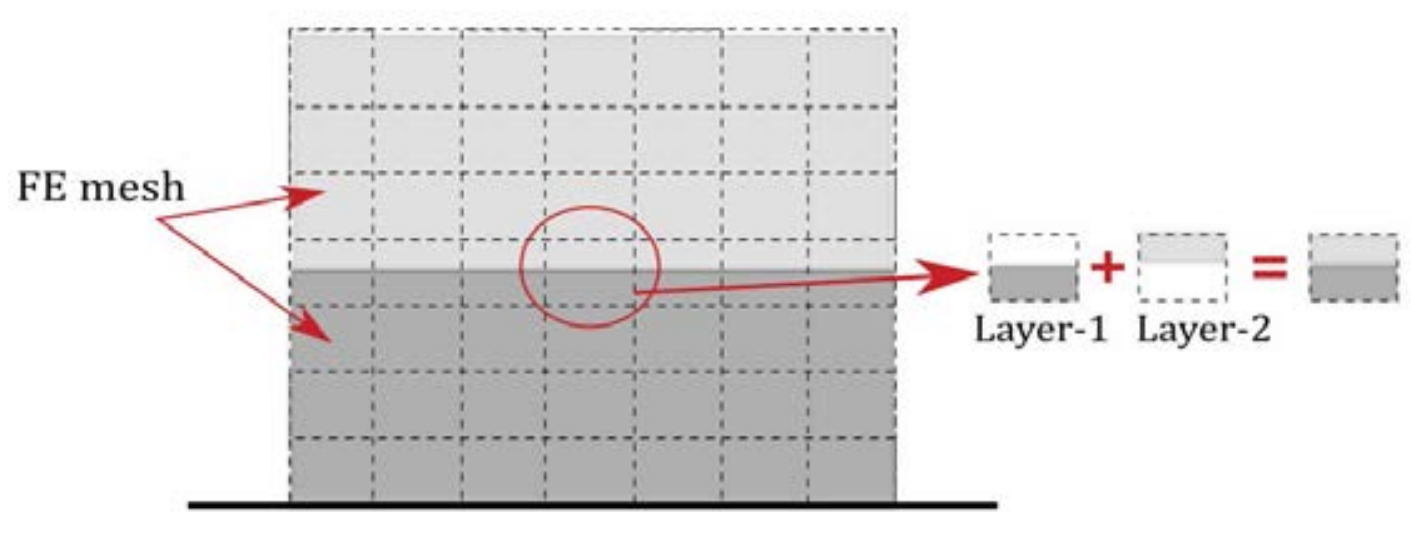

Figure 2: 3D Concrete printed layers and the FE mesh

This has been addressed by introducing a novel modeling approach, applying the FE while considering a pseudo density approach similar to the soft-killing approach in topology optimization, for more detail, see [9].

In addition to the above-mentioned modeling procedure, as mentioned in the previous section uncertainties arising in the model description (geometry, material properties, or loading) are also included by utilizing structural reliability analysis.

Numerical simulation is performed using the commercially available programing language MATLAB, and UQLab (which is an open-source scientific module, a framework for Uncertainty quantification developed at ETH Zurich), to investigate the buildability of 3D concrete printing wall structures during the printing process. 


\subsection{Numerical example}

The wall is modeled as a two-dimensional structure and constructed in a layer-wise process to perform failure analyses. Finite Element analysis with 4-node bi-linear finite elements is applied. The boundary condition considered is the bottom layer fixed as a result of friction on the printed bed [17]. The geometry of the numerical simulation includes wall width $\mathrm{w}=$ $43.5 \mathrm{~mm}$, wall-length $\mathrm{L}=1000 \mathrm{~mm}$, the thickness of each layer $\mathrm{t}=10 \mathrm{~mm}$, the Poisson ratio of the printing material (which is assumed to be constant during the curing process) taken as 0.3 . The analysis was limited to a number of 17 layers to avoid the elastic buckling according to a parametric model developed by Suiker et al. [2]. Deterministic uniform load configuration is used only considering the self-weight as a result of the layer-wise production process and activated as the printing progresses.

\subsubsection{Numerical simulation of the compressive failure}

The temporal changes and the randomness of the compressive strength are represented by random variables [30], generated from the normal distribution [25,26,27]. This random field is characterized statistically by three parameters defining its first moments, the mean, the standard deviation, and the correlation length. Therefore, results obtained from Eq. (2) are taken as the mean value for each Finite Elements(FE), for this process one of the discretization methods i.e. midpoint method is used [16].

In particular, this numerical simulation is focused on buildability (part of overall failure probability) of the 3D printed concrete wall by varying one of the printing process parameter i.e printing velocity. Also, different values of the correlation length have been investigated in the numerical simulation.

The mean and standard deviation can conveniently be combined in terms of the dimensionless coefficient of variation, taking the result from Wolfs et al. [4], to be 0.168 . For example, for printing velocity of $1.4 \mathrm{~m} / \mathrm{mm}$, the temporal development of compressive strength at the bottom layer is described as a Gaussian random field with a mean based on Eq. (2) is $7.8 \mathrm{kPa}$ and the standard deviation is 1.31 . For all the other layers temporal development of compressive strength can be calculated similarly.

In this research, a "Markovian" correlation function is used where the spatial correlation is assumed to decay exponentially with distance [28]. It has the form of:

$$
\rho_{(\tau)}=\exp \left(\frac{-2\left|\tau_{\mathrm{ij}}\right|}{\theta}\right)
$$

where $\theta$ is the correlation length and $\tau_{\mathrm{ij}}$ is the separation distance between two finite elements.

Realizations of the random compressive strength fields are produced using covariance matrix decomposition. It is a direct method of producing a homogeneous random field [36]. A covariance matrix is formulated for a single layer, then the covariance matrix is decomposed into a lower and upper triangular matrix via a LU Decomposition, to generate correlated normally-distributed random variables for each layer.

Additionally, the layer-wise production process of the concrete structure is included in the FE model while considering a pseudo-density approach [9].

$$
\sigma_{y}(x)=\sigma_{y, \min }+\rho^{\mathrm{P}}(\mathrm{x})\left(\sigma_{\mathrm{y}}-\sigma_{\mathrm{y}, \min }\right)
$$


Where $\sigma_{\mathrm{y}}(\mathrm{x})$ denotes the resulting compressive strength of the concrete, $\sigma_{\mathrm{y}, \min }>0$ is a lower bound to avoid zero entries, $\sigma_{\mathrm{y}}$ refers to the nominal compressive strength, spatiallyvarying pseudo-density $\rho(x) \in[0,1]$, and $p>1$ denotes a power-law correlation that is implemented to achieve density values closer to the lower and upper bounds of the design variables.

Based on Wolfs et al. [4] experimental result concrete density is taken as a random variable with normal distribution [25,26,27], the mean value of $2020 \mathrm{~kg} / \mathrm{m}^{3}$, and the coefficient of variation is $1.96 \%$ for the determination of the compressive stress.

To generate the random field for the concrete density, here also the Markovian correlation function Eq. (5) and the covariance matrix decomposition are applied. Correspondingly, material density is modeled considering a pseudo-density approach.

$$
\rho_{c}(x)=\rho_{c, \text { min }}+\rho^{P}(x)\left(\rho_{c}-\rho_{c, \text { min }}\right)
$$

where $\rho_{\mathrm{c}}(\mathrm{x})$ denotes the resulting concrete density, $\rho_{\mathrm{c}, \min }(\mathrm{x})>0$ is a lower bound to avoid zero entries, $\rho_{\mathrm{c}}$ is the nominal concrete density, $\rho$ and $\mathrm{p}$ are similar to Eq.(6).

In a random field, the value assigned to each cell or finite element, in this case, is itself a random variable, thus the mesh which has 1050 finite elements, contains 1050 random variables. For both the compressive strength and the compressive stress for each analysis,3000 realizations were performed with input shown in Table 1 , by varying the printing velocity $\left(v_{\mathrm{p}}\right)$ and Correlation length $(\theta)$.

\begin{tabular}{ll}
\hline Parameter & Values considered \\
\hline$v_{\mathrm{p}}(\mathrm{m} / \mathrm{min})$ & $0.7,1.4,2.1,3$ \\
$\theta(\mathrm{m})$ & $0.1,1,10,100$ \\
\hline
\end{tabular}

Table 1: Parameters varied in the numerical example while holding the other parameters constant.

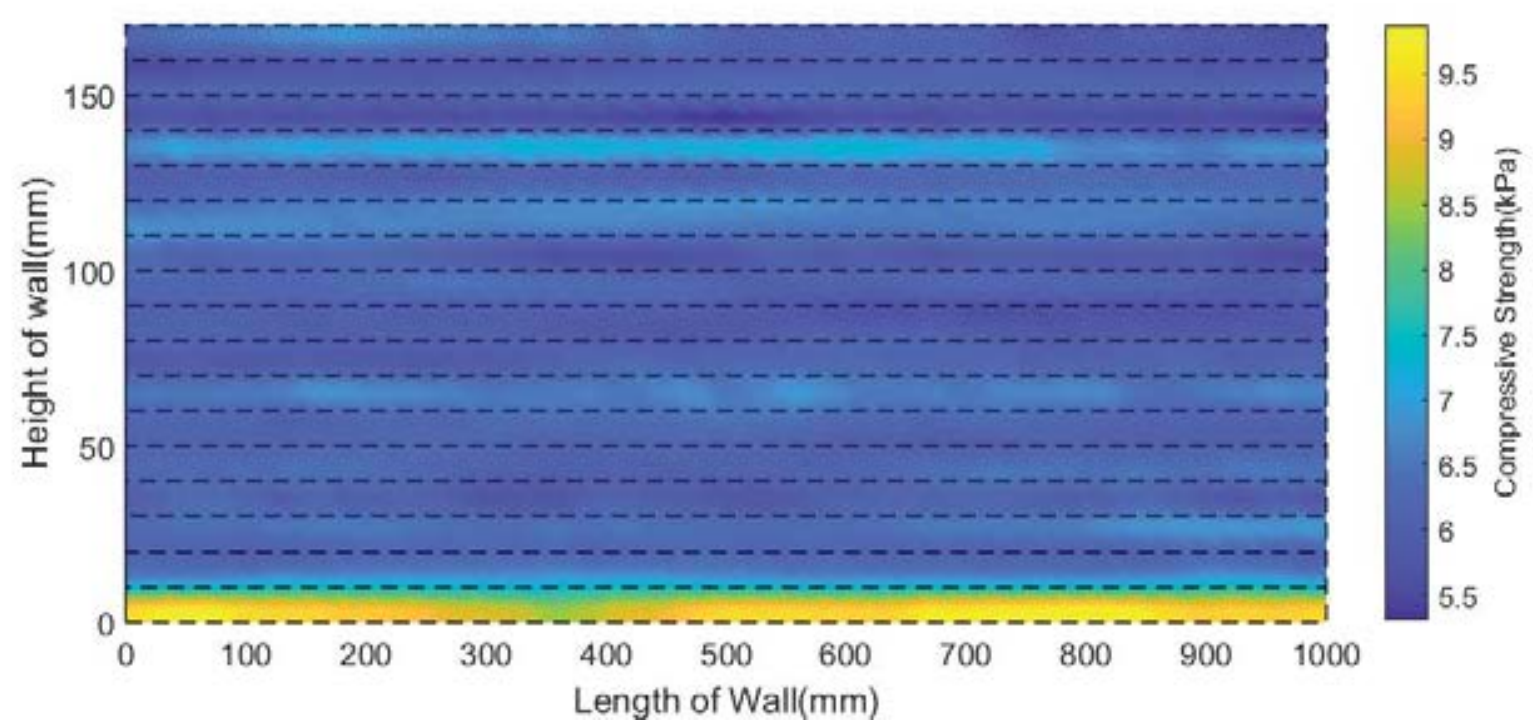

Figure 3: Single realization of the compressive strength for the printing velocity of $2.1 \mathrm{~m} / \mathrm{mm}$ and correlation length $10 \mathrm{~m}$.

A single realization of the compressive strength is shown in Fig.3, both the randomness and time dependency are observed, and the effect of the correlation length is also included in 
the realization. Correlation between the properties of the neighboring freshly printed elements is noticed.

The strength-based failure of the structure is studied by comparing the temporal evolution of the compressive yield stress with the increasing hydrostatic pressure caused by subsequently placed concrete layers (compressive stress). Gravity-induced stresses increase with the height of the printed product, whereby the maximum stress values occur in the bottom layer. For each realization, the mean and standard deviation is taken to perform the failure probability at the bottom layer.

Combining the written MATLAB script and UQLab, the result for the printing velocity of $2.1 \mathrm{~m} / \mathrm{min}$ is shown in Fig. 4 .

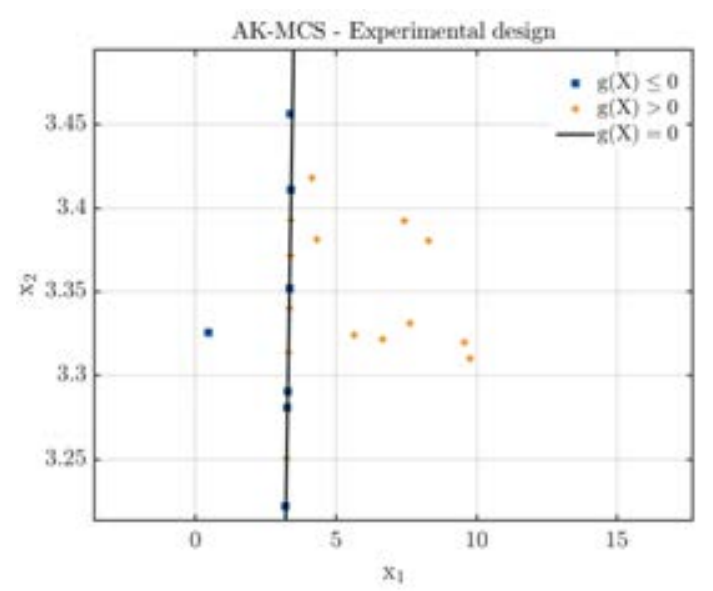

(a)

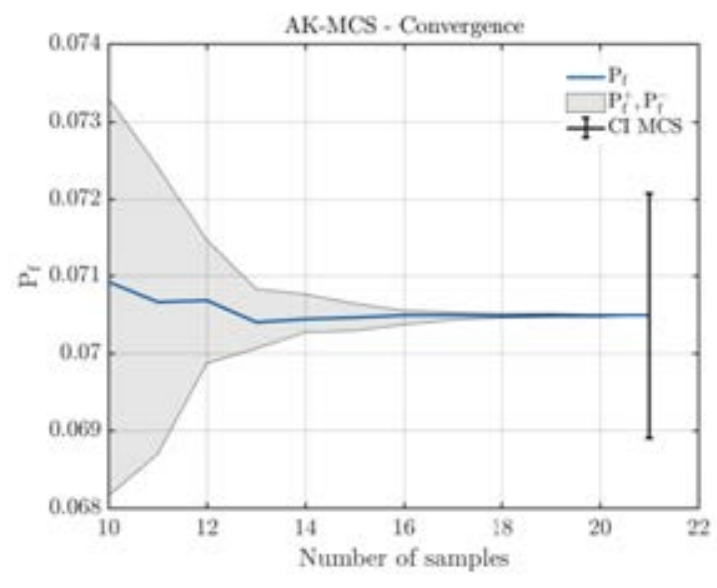

(b)

Figure 4: UQLab output for Adaptive Kriging Monte Carlo Simulation printing velocity of $2.1 \mathrm{~m} / \mathrm{min}$ and correlation length of $0.1 \mathrm{~m}$.

Fig.4 shows graphical visualization of the convergence of the AK-MCS analysis (Fig.4b), a Kriging surrogate model from a small initial sampling of the input vector produces an experimental design iteratively refined close to the currently estimated limit-state the surface $\mathrm{g}(\mathrm{x})=$ 0 (Fig.4a).

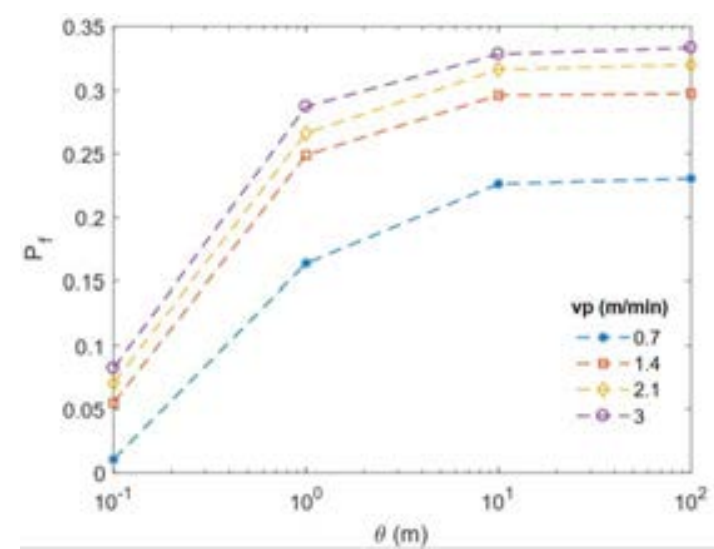

(a)

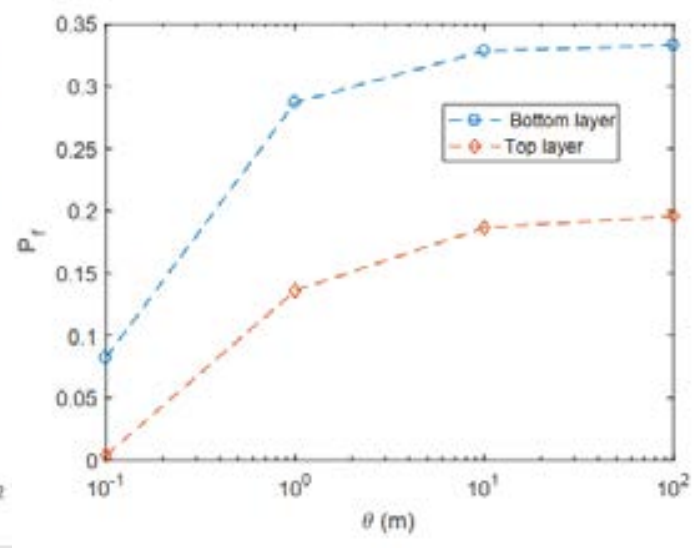

(b)

Figure 5: Effect of printing velocity and correlation length on the Probability of failure of compressive strength. 
The numerical simulation output, see Fig.5a, indicates that with increasing printing velocity, the probability of failure of the buildability of the printed wall increase. This fact was expected because the $3 \mathrm{D}$ concrete printing process is a time-dependent process. Increasing the printing velocity means lowering the curing time, which also results in a lower compressive strength. Another important observation from Fig.5a is the influence of spatial correlation length on the probability of failure. Different correlations cause a large difference in results. If the spatial correlation length for selected printing velocity increases, then the probability of failure also increases.

Furthermore, it can be observed the probability of failure of the top layer is different. The reasons for that are: (1) for the 3D printed wall the concrete age at the top layer is smaller compared to the other layers which affect the compressive strength and (2) to check the accuracy of the numerical simulation. Fig. 5b shows that the probability of failure is higher at the bottom than the other layers as expected even if the curing time for the top layer is smaller than the bottom layer but it is subjected to higher stress, the aforementioned result is for $3 \mathrm{~m} / \mathrm{min}$ printing velocity.

\section{CONCLUSION}

A numerical modeling procedure of a 3D concrete printed (3DCP) wall is proposed, in which uncertainties implemented via the Stochastic Finite Element Method (SFEM) and a spatially varying pseudo-density approach are considered. To minimize the computational cost, Adaptive Kriging Monte Carlo Simulation (AK-MCS) is utilized.

The aforementioned method enables to predict the probability of failure of the 3D printed concrete wall. The influence of the printing velocity and the spatial correlation length on the probability of failure was studied. For a simple 2D example of a wall, it was shown clearly that the influence of spatial correlation length on the probability of failure is significant. Different correlation lengths cause a difference in the results. However, in practice correlation lengths are often assumed based on literature and these assumed values for the correlation length of concrete properties are not consistent with each other [32]. The suggested values for the correlation length could be the initial values for further investigations.

In the future, additional failure mechanisms, the influence of additional mechanical properties, and process parameters will be studied. It will give rise to a more realistic 3DCP model.

\section{ACKNOWLEDGMENT}

The work has been financially supported by different institutions which are highly acknowledged. Among them are DAAD (Ethiopian - German Exchange of Ph.D. candidates), DFG (German Research Foundation) priority program 1886 "Polymorphic uncertainty modeling for the numerical design of structures" and the Federal State of Thuringia, Germany.

\section{REFERENCES}

[1] R. A. Buswell, W. R. Leal de Silva, S. Z. Jones, and J. Dirrenberger. 3d printing using concrete extrusion: A roadmap for research. Cement and Concrete Research, 112:37-49, 2018 . 
[2] Suiker, A.: Mechanical performance of wall structures in 3D printing processes: Theory, design tools, and experiments. International Journal of Mechanical Sciences 137, 145170,2018 .

[3] Wolfs, R. J. M. Experimental characterization and numerical modeling of 3D printed concrete: controlling structural behavior in the fresh and hardened state. Eindhoven: Technische Universiteit Eindhoven,2019

[4] R. J. M. Wolfs, F. P. Bos, and T. A. M. Salet. Early age mechanical behaviour of 3d printed concrete: Numerical modeling and experimental testing. Cement and Concrete Research, 106:103-116, 2018.

[5] Roussel, N.: Cement and Concrete Research Rheological requirements for printable concretes. Cement and Concrete Research, 1-10 (2018). 2018.

[6] Marchon, D., Kawashima, S., Bessaies-Bey, H., Mantellato, S., Ng, S.: Hydration and rheology control of concrete for digital fabrication: Potential admixtures and cement chemistry. Cement and Concrete Research 112: 96-110,2018.

[7] Le, T.T., Austin, S.A., Lim, S., Buswell, R.A., Law, R., Gibb, A.G., Thorpe, T.: Hardened properties of high-performance printing concrete. Cement and Concrete Research 42:558-566,2012.

[8] Perrot, A.: 3D Printing of Concrete. ISTE Ltd and John Wiley \& Sons, Inc, Great Britain and the United States by, first edn,2019.

[9] Mengesha M., Schmidt A., Göbel L., Lahmer T. Numerical Modeling of an ExtrusionBased 3D Concrete Printing Process Considering a Spatially Varying Pseudo-Density Approach. Second RILEM International Conference on Concrete and Digital Fabrication. DC 2020. RILEM Bookseries, vol 28, 2020. Springer International Publishing.

[10] Arregui-Mena, J. D., Margetts, L., \& Mummery, P. M. Practical Application of the Stochastic Finite Element Method. Archives of Computational Methods in Engineering (Vol. 23),2016.

[11] Brenner, C. E., \& Bucher, C. G. Stochastic response of uncertain systems. Archive of Applied Mechanics, 62(8), 507-516,1992.

[12] Gomes, H. M., \& Awruch, A. M. Reliability of reinforced concrete structures using stochastic finite elements. Engineering Computations (Swansea, Wales), 19(7-8), 764786,2002 .

[13] Sudret, B., \& Kiureghian, A. Der.Stochastic finite element methods and reliability. Ucb/Semm-2000, (October), 189,2000.

[14] Andrea, B., \& Brunel, J. F. SLOVAK UNIVERSITY OF TECHNOLOGY IN BRATISLAVA Bratislava 2013.

[15] Aldosary, M., Wang, J., \& Li, C. Structural reliability and stochastic finite element methods: State-of-the-art review and evidence-based comparison. Engineering Computations (Swansea, Wales), 35(6), 2165-2214,2018.

[16] Bergman, L. A., Shinozuka, M., Bucher, C. G., Sobczyk, K., Dasgupta, G., Spanos, P. D., ... Zhang, R. A state-of-the-art report on computational stochastic mecha ics. Probabilistic Engineering Mechanics, 12(4), 197-321,1997. 
[17] Gordon A. Fenton and D. V. Griffiths. Risk Assessment in Geotechnical Engineering. John Wiley \& Sons, 2008.

[18] Papadrakakis, M., \& Papadopoulos, V. Robust and efficient methods for stochastic finite element analysis using Monte Carlo simulation. Computer Methods in Applied Mechanics and Engineering, 134(3-4), 325-340,1996.

[19] Sataloff, R. T., Johns, M. M., \& Kost, K. M. (n.d.). Reliability-Based Design in Geotechnical Engineering. Taylor \& Francis e-Library, 2008.

[20] R. A. Buswell, W. R. Leal de Silva, S. Z. Jones, and J. Dirrenberger. 3D printing using concrete extrusion: A roadmap for research. Cement and Concrete Research, 112(May):37-49, 2018.

[21] J. van der Putten, G. de Schutter, and K. van Tittelboom. The effect of print parameters on the (micro)structure of $3 \mathrm{~d}$ printed cementitious materials. In Timothy Wangler and Robert J. Flatt, editors, First RILEM International Conference on Concrete and Digital Fabrication -Digital Concrete 2018, pages 234-244, Cham, 2019. Springer International Publishing.

[22] Marelli, S., Schöbi, R., Sudretm, B.: UQLab user manual - Structural reliability (Rare event estimation), Report \# UQLab-V1.3-107,2019.

[23] Wolfs, R.J., Suiker, A.S.: Structural failure during extrusion-based 3D printing processes. International Journal of Advanced Manufacturing Technology 104(1-4), 565584,2019 .

[24] Echard, B., Gayton, N., \& Lemaire, M. AK-MCS: An active learning reliability method combining Kriging and Monte Carlo Simulation. Structural Safety, 33(2), 145154.,2011.

[25] De Araujo, J.M.: Probabilistic analysis of reinforced concrete columns. Advances in Engineering software 32, 871-879,2001.

[26] El-Reedy, M.A.: Reinforced Concrete Structural Reliability. CRC Press ,2012.

[27] Seo, D., Shin, S., Han, B.: Reliability-based structural safety evaluation of reinforced concrete members. Journal of Asian Architecture and Building Engineering 9(2), 471478,2010 .

[28] Vanmarcke, B. E., Asce, M., \& Grigoriu, M. Stochastic finite element analysis of simple beams, 109(5), 1203-1214,1984.

[29] Bottenbruch, H., Pradlwarter, H.J., Schuëller, G.I.: The influence of spatial correlation of concrete strength on the failure probabilities of reinforced concrete chimneys. Materials and Structures 22(4), 255-263,1989.

[30] Vanmarcke, E., Shinozuka, M., Nakagiri, S., Schuëller, G. I., \& Grigoriu, M. Random fields and stochastic finite elements. Structural Safety, 3(3-4), 143-166,1986.

[31] Yang, Y., Peng, J., Zhang, J., \& Cai, C. S. . A new method for estimating the scale of fluctuation in reliability assessment of reinforced concrete structures considering spatial variability. Advances in Structural Engineering, 21(13), 1951-1962,2018.

[32] Criel, P., Caspeele, R., \& Taerwe, L. Bayesian updated correlation length of spatial concrete properties using limited data. Computers and Concrete, 13(5), 659-677,2014 
[33] Griffiths, D. V., \& Lane, P. A. Slope stability analysis by finite elements. Geotechnique, 49(3), 387-403,1999.

[34] Maiti, \& Bidinger. CISM COURSES AND LECTURES. Journal of Chemical Information and Modeling, 53(9), 1689-1699,1981.

[35] Giulio Maier - Milan, Jean Salençon - Palaiseau, \& Wien, W. S.-.cism courses and lectures. the International Center for Mechanical Sciences, SpringerWien NewYork,2007.

[36] De Schutter, G., Lesage, K., Mechtcherine, V., Nerella, V., Habert, G., Agusti-Juan, I.: Vision of 3D printing with concrete $\mid$ Technical, economic and environmental potentials. Cement and Concrete Research 112, 25-36,2018. 